\title{
Sesame allergy: current perspectives
}

This article was published in the following Dove Press journal:

Journal of Asthma and Allergy

27 April 2017

Number of times this article has been viewed

\author{
Adil Adatia' \\ Ann Elaine Clarke ${ }^{2}$ \\ Yarden Yanishevsky ${ }^{3}$ \\ Moshe Ben-Shoshan ${ }^{4}$ \\ 'Department of Medicine, University \\ of Alberta, Edmonton, ${ }^{2}$ Division \\ of Rheumatology, Department \\ of Medicine, Cumming School of \\ Medicine, University of Calgary, \\ Calgary, ${ }^{3}$ Section of Allergy and \\ Clinical Immunology, Department \\ of Pediatrics, University of Alberta, \\ Edmonton, $A B,{ }^{4}$ Division of Pediatric \\ Allergy and Clinical Immunology, \\ Department of Pediatrics, Montreal \\ Children's Hospital, McGill University \\ Health Centre, Montreal, QC, Canada
}

\begin{abstract}
Sesame is an important global allergen affecting $\sim 0.1 \%$ of the North American population. It is a major cause of anaphylaxis in the Middle East and is the third most common food allergen in Israel. We conducted a systematic review of original articles published in the last 10 years regarding the diagnosis and management of sesame allergy. Skin prick testing appears to be a useful predictor of sesame allergy in infants, although data are less consistent in older children and adults. The diagnostic capacity of serum-specific immunoglobulin $\mathrm{E}$ is poor, especially in studies that used oral food challenges to confirm the diagnosis. Doubleblind, placebo-controlled food challenge thus remains the diagnostic gold standard for sesame allergy. The cornerstone of sesame allergy management is allergen avoidance, though accidental exposures are common and patients must be prepared to treat the consequent reactions with epinephrine. Novel diagnostic and treatment options such as component-resolved diagnostics, basophil activation testing, and oral immunotherapy are under development but are not ready for mainstream clinical application.
\end{abstract}

Keywords: sesame allergy, skin prick testing, specific IgE, component-resolved diagnostics, epinephrine autoinjector

\section{Introduction}

Sesame (Sesamum indicum) is a seed native to the Middle East and Africa, where it has been cultivated as an oilseed crop for $>3000$ years. It is traditionally consumed as a paste called tahini or as a sweet called halva and is also used as toppings on breads and crackers, particularly in Western countries. The population prevalence estimates of self-reported sesame allergy in the $\mathrm{USA}^{1}$ and Canada ${ }^{2,3}$ range from $0.1 \%$ to $0.2 \%$. Studies report prevalence estimates of $0.1 \%$ in Canadian children ${ }^{3}$ and up to $0.8 \%$ among children in Australia. ${ }^{4}$ The rate of sesame-induced anaphylaxis varies significantly by region, with much higher rates reported in the Middle East compared to those in North America. Sesame is reported as the second most common food to cause anaphylaxis in Israeli children, accounting for $43 \%$ of cases $^{5}$ and the third most common food to cause anaphylaxis in Saudi Arabia, ${ }^{6}$ but it was responsible for only $2.8 \%$ of food-induced anaphylaxis cases in Canadian children. ${ }^{7}$ Despite its relatively low prevalence in North America, sesame allergy is important to recognize given that sesame allergy is rarely outgrown and the risk of accidental reactions is high. ${ }^{8-10}$

In this systematic review, we assess the literature published in the last 10 years regarding sesame allergy with a focus on the diagnosis and management of this important global allergen.
Correspondence: Moshe Ben-Shoshan Division of Allergy and Clinical Immunology, Department of Pediatrics, Montreal Children's Hospital, I00I Decarie, Montreal, QC H4A 3JI, Canada

Tel + I 5144124400 ext 24470

$\mathrm{Fax}+|5| 4228$ II97

Email moshebenshoshan@gmail.com 


\section{Methods}

We (MB-S and AA) searched the PubMed and EMBASE databases for original scientific studies pertaining to the diagnosis and management of sesame allergy. We used the search criteria "sesame" AND "allergy" and then limited the results to articles published between November 1, 2006, and November 1, 2016, that were written in English and French. The abstracts of the resulting papers were then reviewed and those relevant to the diagnosis and management of sesame allergy were included in the systematic review and have been summarized in Tables 1 and 2. The initial PubMed and EMBASE database queries yielded a total of 268 articles, which was reduced to 162 after applying the above filters and then further narrowed to 30 articles after the 162 abstracts were assessed for relevancy to the systematic review (Figure 1).

\section{Diagnosis}

\section{Skin prick testing (SPT)}

SPT is a key component in the diagnosis of food allergy. In 2013, the HealthNuts study, a population-based, longitudinal food allergy study in Melbourne, Australia, examined the diagnostic performance of sesame SPT in infants aged 11 to 15 months. Sesame SPT data were available in 103 patients who had a conclusive oral food challenge (OFC). The 95\% positive predictive value (PPV) threshold was $8 \mathrm{~mm}$ or greater ( $95 \%$ confidence interval [CI], 5-9 mm), which corresponded to a sensitivity and specificity of $48 \%$ and $99 \%$, respectively. However, the PPV of a wheal $3 \mathrm{~mm}$ or greater, a threshold commonly used to define a "positive" SPT, was $\sim 58 \% .{ }^{11}$ Receiver operator characteristic (ROC) curve analysis showed the test was highly accurate with an area under the curve (AUC) of 0.92. Similarly, in an Israeli cohort of 16 children and adults who underwent OFC because of a clinical history suggestive of sesame allergy, a positive SPT (which was defined as wheal size equal to or greater than the wheal size of the histamine control, though all but one patient had a wheal diameter of $3 \mathrm{~mm}$ or greater) had a PPV of $88 \%$, supporting the diagnostic value of sesame SPT. ${ }^{10}$

However, other studies have not strongly supported the diagnostic utility of sesame SPT. Permaul et al found that a positive SPT (wheal more than $3 \mathrm{~mm}$ greater than negative control) had $71 \%$ sensitivity, $58 \%$ specificity, $31 \%$ PPV, and $88 \%$ negative predictive value (NPV) using OFC as the gold standard in a retrospective study of children aged 2-12 years. ROC curve analysis showed lower accuracy compared to the HealthNuts study with an AUC of 0.67 , and a $95 \%$ PPV threshold could not be calculated. ${ }^{12}$ The same research group obtained similar results in a second retrospective chart review of patients aged 6.6 months to 19.6 years, in which a positive SPT had $43.5 \%$ sensitivity, $64.3 \%$ specificity, $14.3 \%$ PPV, and $89.3 \%$ NPV, though in this study, the diagnostic performance measures were calculated using a convincing reaction history as the gold standard. ${ }^{13}$ The apparent inconsistent performance of SPT in the literature is likely related to differences in the populations studied, particularly with respect to age and geographical area. In addition, Permaul et al ${ }^{12}$ included primarily peanut allergic patients who had positive sesame allergy screening tests, which may have contributed to the low rate of positive OFCs that they observed. Those positive tests could represent clinically inconsequential cross-reactivity between peanut-specific immunoglobulin E (IgE) and sesame, a possibility that is supported by the significant homology that exists between sesame and peanut allergens.

\section{Skin-prick-prick (SPP) testing}

SPP testing, which is an SPT that uses sesame seeds, oil, or tahini instead of a commercial extract, may also be useful when investigating possible sesame allergy. Sesame seeds are typically crushed and suspended in saline for ease of administration, ${ }^{14}$ whereas sesame oil and tahini can be applied to the skin undiluted. ${ }^{15}$ The relative quantities of the major sesame allergens in sesame seed, oil, and tahini have not been systematically studied, though the preparation procedures used to make sesame oil and tahini, including roasting, are not thought to reduce their allergenicity. ${ }^{15,16}$

A British group evaluated the diagnostic performance of SPP testing using tahini in a retrospective cohort of 79 children. They found that a $2 \mathrm{~mm}$ wheal threshold yielded the best sensitivity (70\%) and specificity $(73 \%) .{ }^{17}$ Sesame SPP testing has also been proposed as a tool to diagnose sesame allergy in patients with negative SPT and serumspecific IgE (sIgE). Della-Torre et $\mathrm{al}^{15}$ reported a case of a 55-year-old male with suspected sesame allergy who had negative SPT and sIgE but positive SPP testing using tahini. Similarly, Barbarroja-Escudero et al evaluated 10 patients with convincing histories of sesame allergy and negative SPT and found that 9 had positive SPP tests using sesame seeds. They posited that cases of sesame allergy with negative SPTs may be accounted for by the absence of lipophilic antigens within commercial sesame extracts due to defatting procedures used during the production process. In their cohort, immunoblotting using each patient's serum demonstrated IgE binding to oleosins, allergenic sesame proteins that are fat soluble. ${ }^{18}$ 


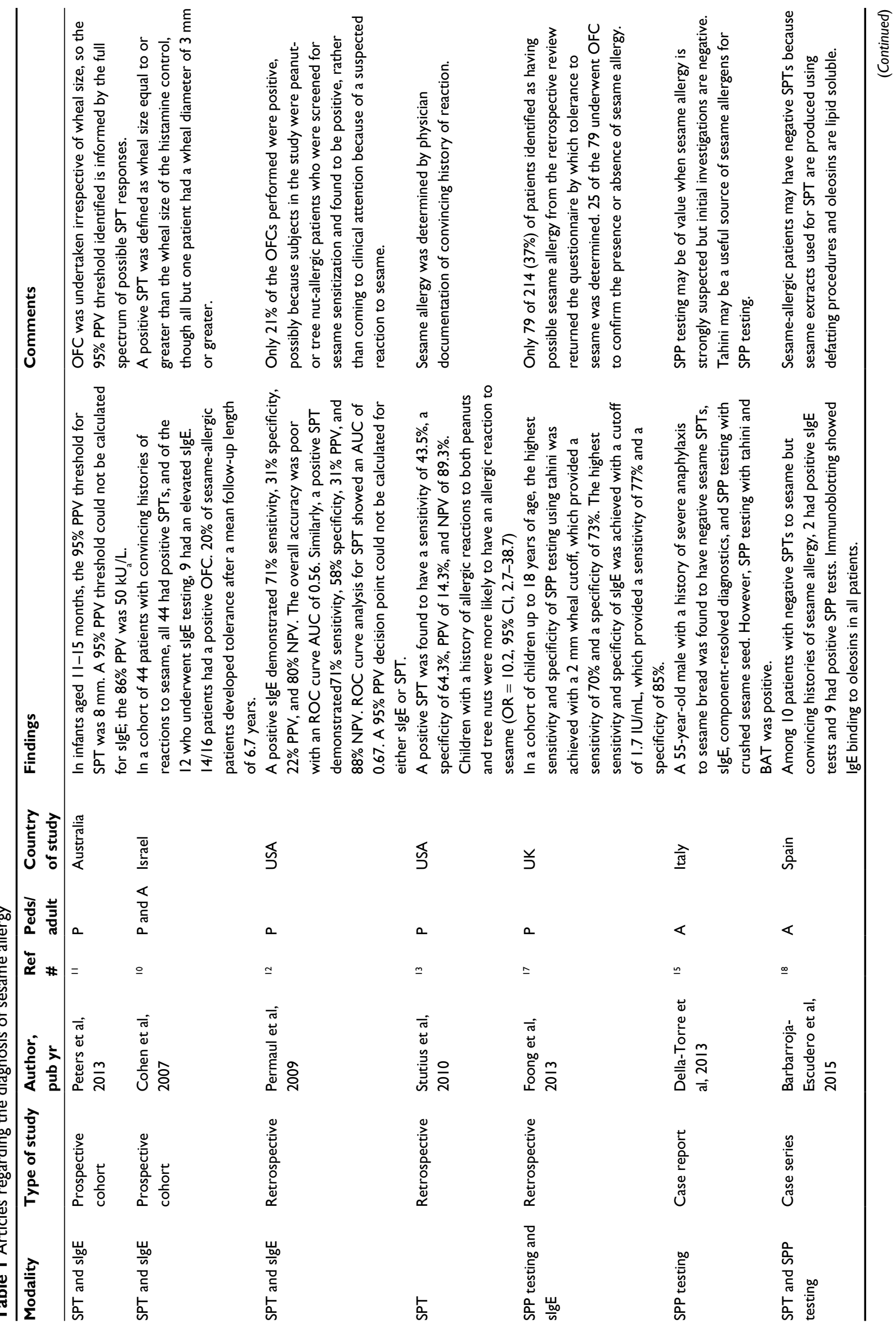




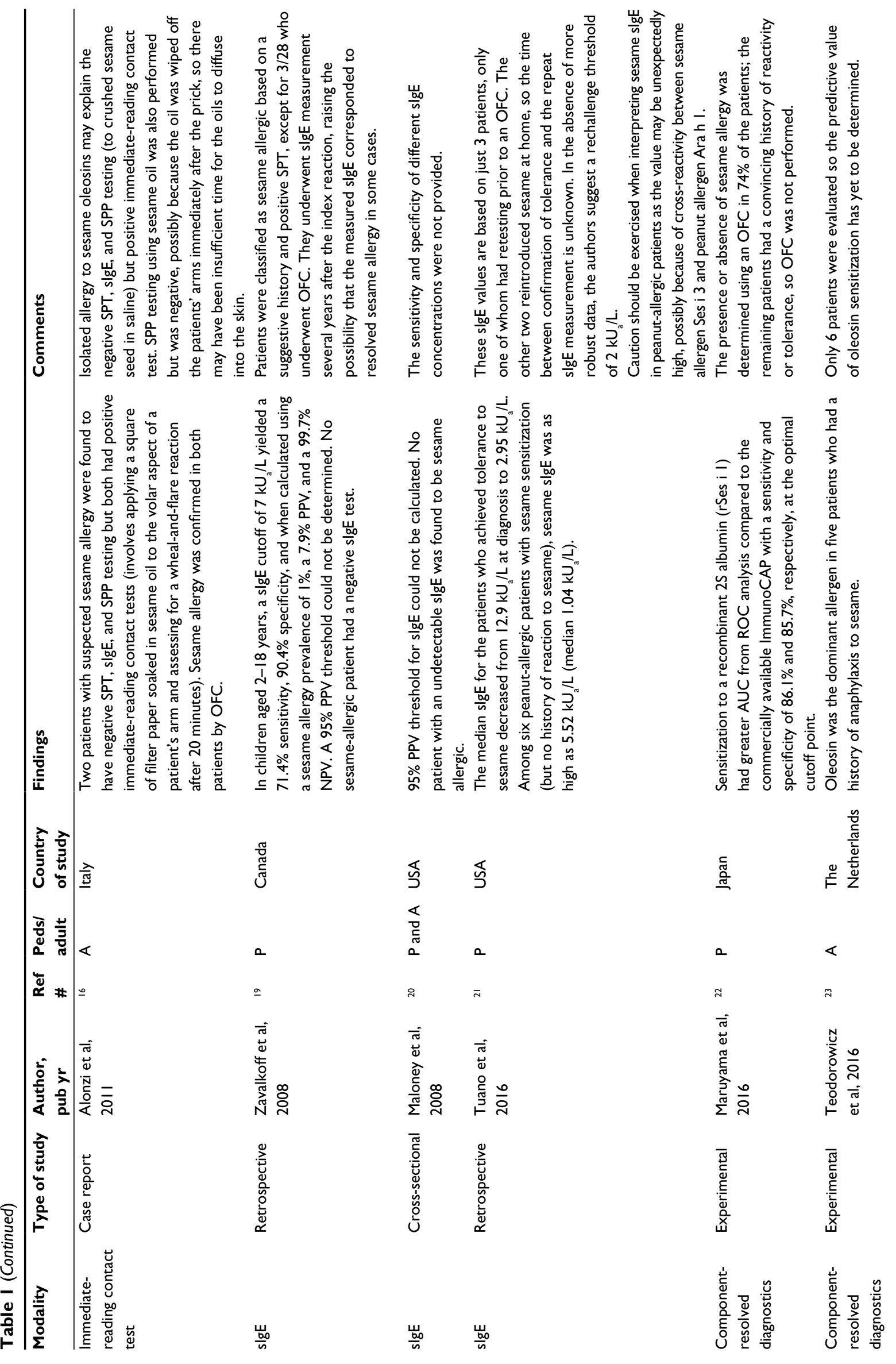




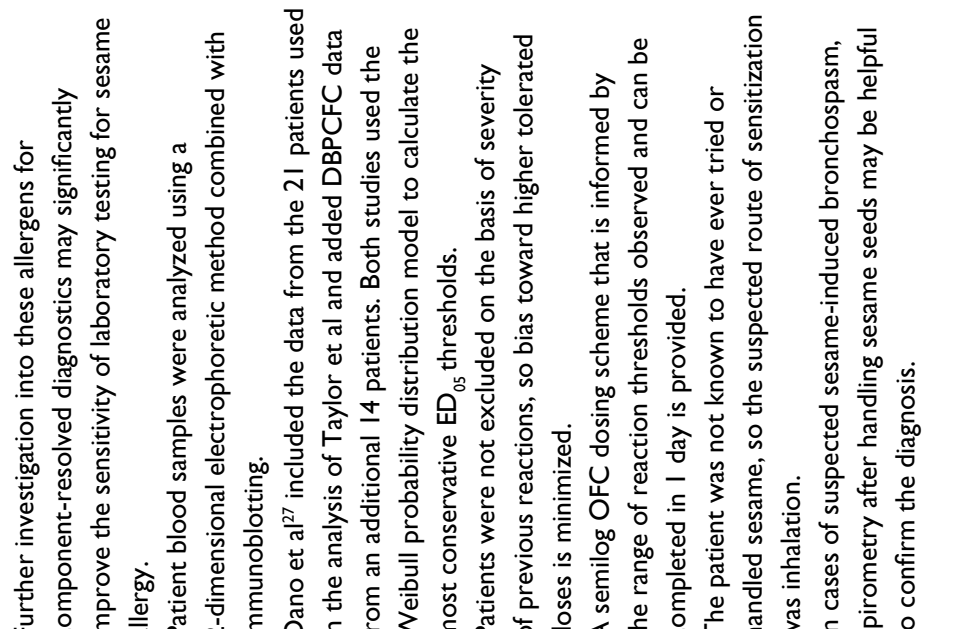

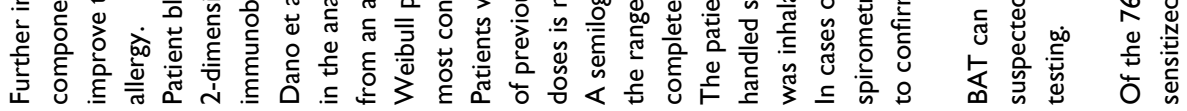
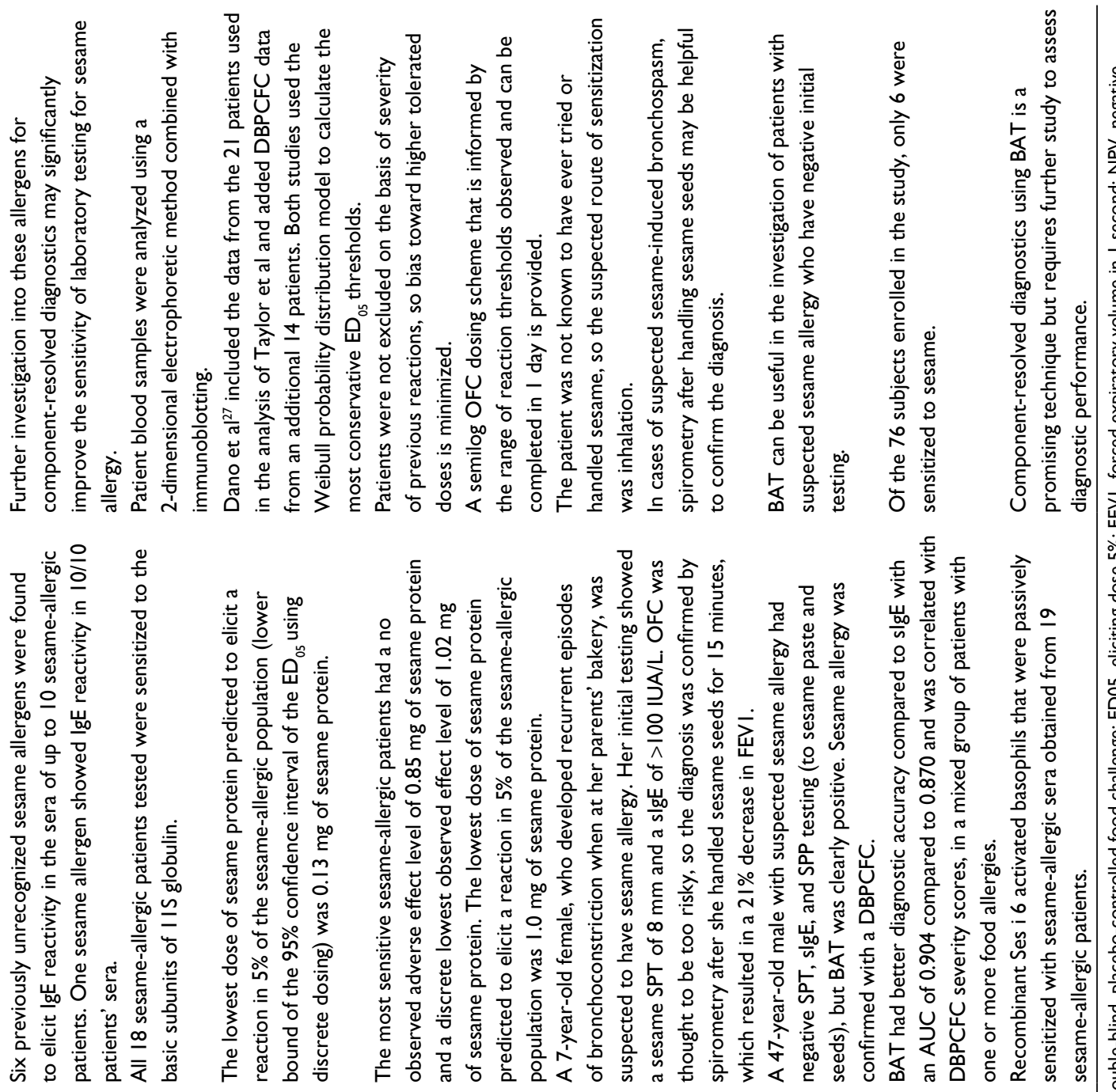

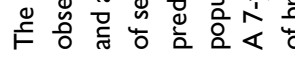
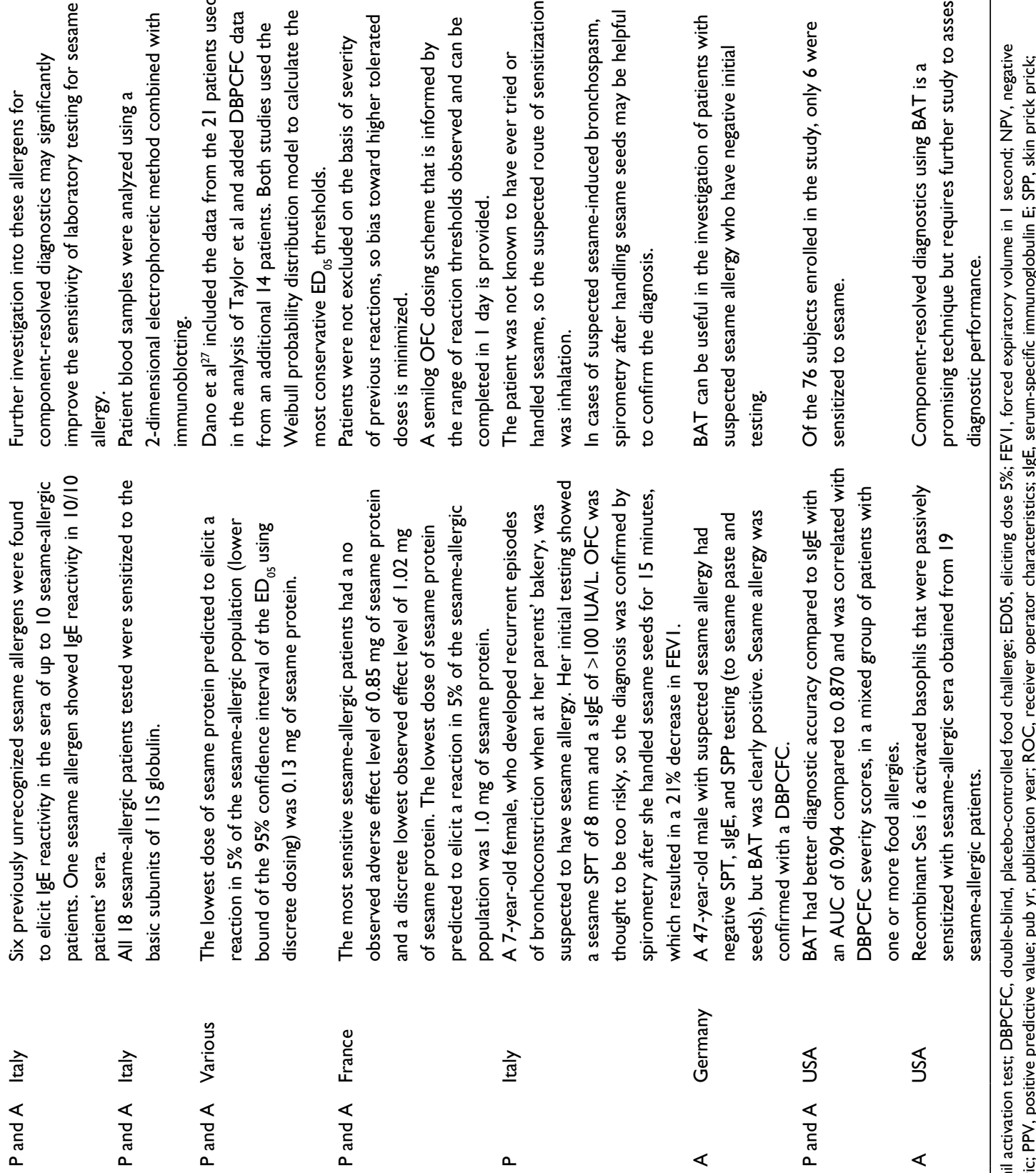

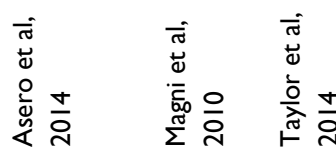

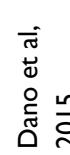

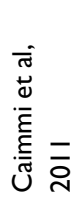

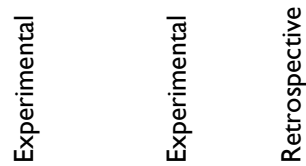

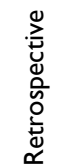

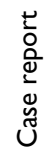

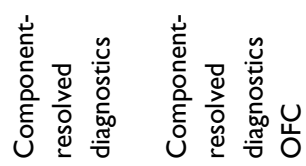

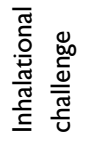
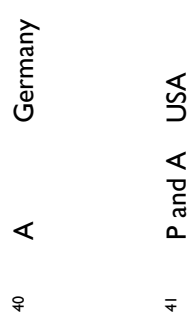

$\overleftrightarrow{\overleftarrow{s}}$

迹

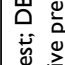

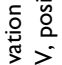

密离

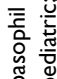

家离

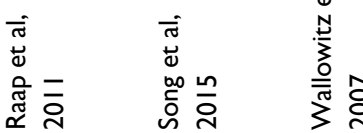<smiles>CCCCCC</smiles>

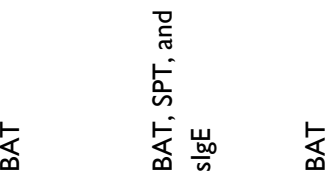




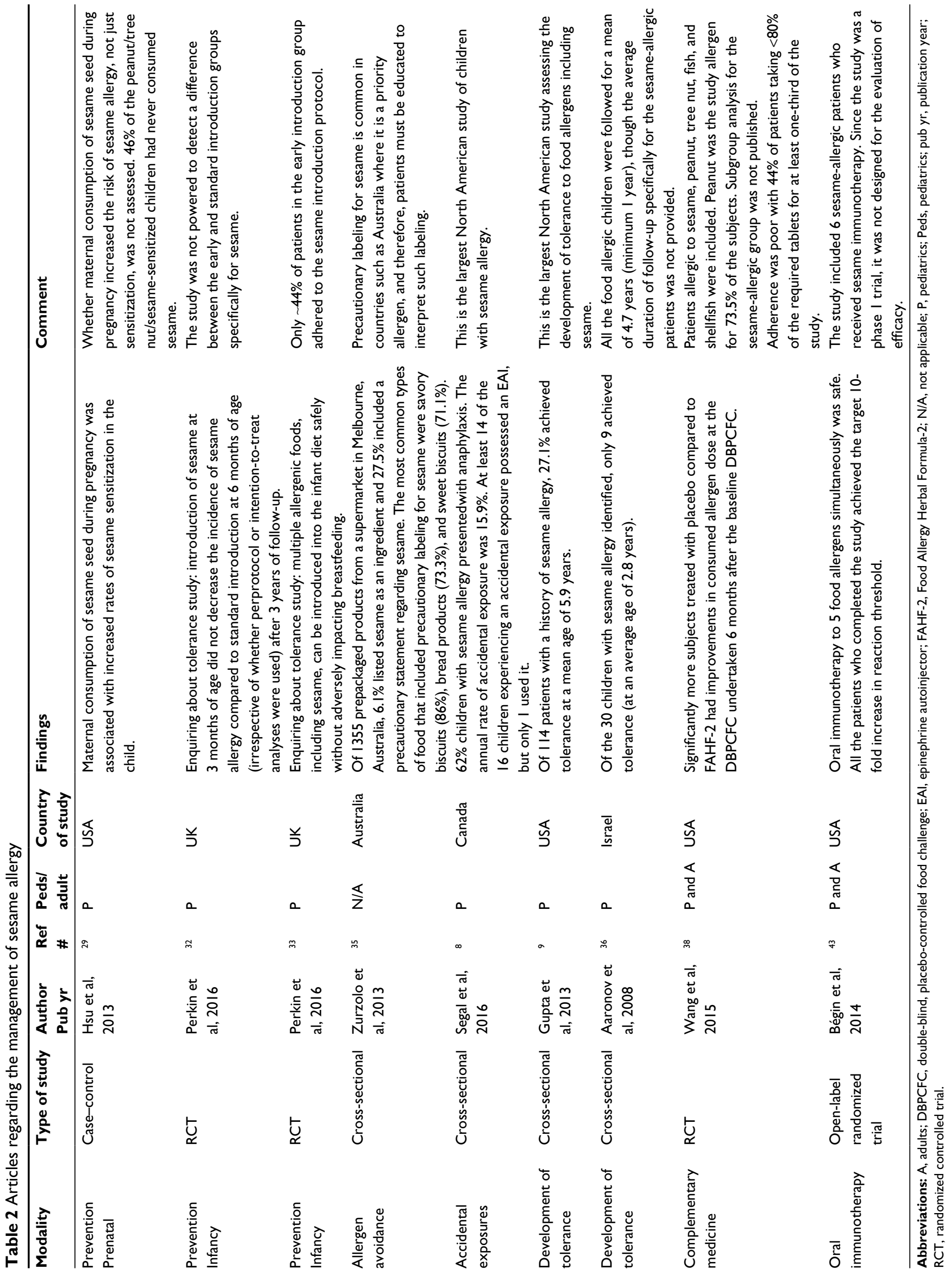




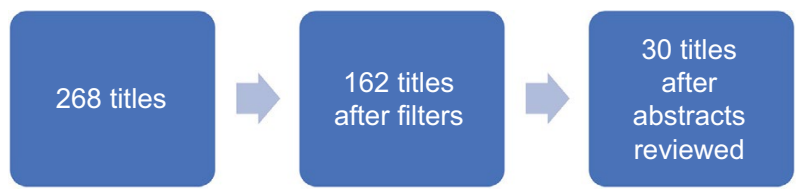

Figure I Results of the systematic literature review using the PubMed and EMBASE databases.

Note: Excluded papers did not pertain to the diagnosis or management of sesame allergy or were reviews rather than original articles.

An Italian group has also described an "immediatereading contact test" for sesame allergy, which involves the application of a square of filter paper soaked in sesame oil to the volar aspect of a patient's arm and assessing for a whealand-flare reaction after 20 minutes. This test was positive in two patients (with OFC-confirmed sesame allergy) who had negative SPT, sIgE, and SPP tests. The authors also attributed their results to the presence of oleosins within sesame oil. ${ }^{16}$

\section{Serum-specific IgE concentration}

Measurement of serum concentrations of $\operatorname{IgE}$ specific to a food antigen (sIgE) is also an important diagnostic tool in the investigation of food allergies. However, two studies suggest that sesame sIgE concentration has poor diagnostic performance. The HealthNuts study described earlier found that the highest PPV threshold that could be calculated was $86 \%$, which corresponded to a $\operatorname{sigE}$ of $50 \mathrm{kU} / \mathrm{L}$ or greater. The diagnostic accuracy of sIgE was low with an AUC of 0.76 on ROC curve analysis. ${ }^{11}$ These results were similar to previously published data from Permaul et al, ${ }_{1}^{12}$ which showed that a positive sIgE test had $71 \%$ sensitivity, $31 \%$ specificity, $22 \%$ PPV, $80 \%$ NPV, and low overall accuracy with an ROC curve AUC of 0.56 .

These results are inconsistent with the data from a British group that evaluated the diagnostic performance of sIgE in a retrospective cohort of 79 children. They found that a relatively low threshold of $1.7 \mathrm{IU} / \mathrm{mL}$ provided a sensitivity of $77 \%$ and specificity $85 \%$, but OFC was only performed in 25 of the patients, so the remaining patients were classified as sesame allergic or nonallergic based on clinical history. ${ }^{17}$ Similarly, Zavalkoff et a ${ }^{19}$ found that, in children aged 2-18 years, a sIgE cutoff of $7 \mathrm{kU} / \mathrm{L}$ yielded a $71.4 \%$ sensitivity and $90.4 \%$ specificity, but again only 3 of the 28 subjects underwent OFC. A decision point for the 95\% PPV could not be determined. Since no sesame-allergic patient had an undetectable sIgE, the authors suggested that sIgE could be useful to exclude rather than diagnose sesame allergy. These findings were corroborated by Maloney et al who also found that all sesame-allergic patients had detectable sIgE concentrations and that the test was not sufficiently predictive to calculate a 95\% PPV threshold, in American children and adults. However, the study was similarly limited by the small number of OFCs performed and by the exclusion of patients with inconclusive clinical histories. ${ }^{20}$ The use of an undetectable sesame sIgE to rule out sesame allergy was not supported by Permaul et al ${ }^{12}$ who, using OFC as the diagnostic standard, reported that $29 \%$ of sesame-allergic patients had SIgE below the detection threshold.

Caution must also be exercised when interpreting sesame sIgE in patients with other preexisting food allergies. Tuano et al showed that sesame $\operatorname{sigE}$ can be as high as $5.52 \mathrm{kU} / \mathrm{L}$ in peanut-allergic patients who tolerate sesame. The suspected mechanism behind elevated sesame sIgE in some peanut allergic patients is cross-reactivity between sesame allergen Ses i 3 and peanut allergen Ara h $1 .{ }^{21}$ Though not yet studied, similar contaminant increases in sesame sIgE may also be found in patients allergic to other foods whose allergens cross-react with sesame allergens.

\section{Component-resolved diagnostics}

Recently, it has been reported that IgE testing to food allergen components can increase the accuracy of specific IgE testing. This method measures the serum concentration of $\operatorname{IgE}$ antibodies to specific allergenic proteins within food and is an area of significant interest to reduce the number of OFCs required. The World Health Organization/International Union of Immunological Societies' Allergen Nomenclature Subcommittee has registered seven sesame allergy components two $2 \mathrm{~S}$ albumins (Ses i 1 and Ses i 2), one vicilin-like 7S globulin (Ses i 3), two oleosins (Ses i 4 and 5), and two 11S globulins (Ses i 6 and Ses i 7). However, to date very few studies have evaluated this technique in sesame allergy. In one study of 92 children sensitized to sesame, sensitization to a recombinant form of the protein $2 \mathrm{~S}$ albumin (rSes i 1 ) had a comparable sensitivity to the commercial assay measuring sIgE (86.1\% for rSes i 1 compared to $83.3 \%$ for the commercial assay) but significantly better specificity $(85.7 \%$ compared to $48.2 \%$ ) for sesame allergy. ${ }^{22}$ Teodorowicz et a ${ }^{23}$ identified the major allergens in six sesame-allergic patients and found that oleosin was the dominant allergen in the five patients who had a history of anaphylaxis to sesame, leading to the hypothesis that sensitization to oleosin may identify a more severe sesame-allergic phenotype.

One of the barriers to the application of componentresolved diagnostics in sesame allergy is that a number of allergenic proteins and protein subunits of sesame have not yet been identified. An Italian group showed that all 10 of the sesame-allergic patients they studied were sensitized to at least one unregistered protein. ${ }^{24}$ Magni et $\mathrm{al}^{25}$ applied a 
2-dimensional electrophoretic method combined with immunoblotting and found that all the sesame-allergic patients studied were sensitized to the basic subunits of $11 \mathrm{~S}$ globulin.

\section{Food challenge}

The gold standard for diagnosing sesame allergy remains the double-blind, placebo-controlled food challenge (DBPCFC), though for practical purposes unblinded OFCs without a placebo control are usually employed. OFC is considered when the history is not convincing or the patient has never been exposed, but the patient tests positive for sesame sensitization by SPT or sIgE. Dano et al used data from 35 DBPCFCs to determine the no observed adverse effect level (NOAEL), defined as the largest amount of food that an individual can ingest without causing an adverse reaction, and the lowest observed adverse effect level (LOAEL), the lowest dose of an allergen ingested that produces an adverse effect. They included data from 21 patients that had already been collated and analyzed by the Voluntary Incidental Trace Allergen Labeling program ${ }^{26}$ and collected data from 14 additional patients. The lowest discrete NOAEL recorded was $0.85 \mathrm{mg}$, and the lowest discrete LOAEL recorded was $1.02 \mathrm{mg}$ of sesame protein. The lowest dose of sesame protein predicted to elicit a reaction in 5\% of the sesame-allergic population (i.e., the eliciting dose $5 \%\left[\mathrm{ED}_{05}\right]$ ) was $1.0 \mathrm{mg}$. Based on these data, an OFC dosing scheme with the following semilog increments was suggested: $0.3,3,30,100,300,1000$, and $3000 \mathrm{mg}$ of sesame protein. ${ }^{27}$

In addition to OFC, sesame inhalation challenges may be useful in specific circumstances. Caimmi et al reported a case of a 7-year-old-female who developed recurrent episodes of bronchoconstriction when at her parents' bakery. Her initial testing showed a sesame SPT of $8 \mathrm{~mm}$ and a sIgE of $>100$ IUA/L. OFC was considered too risky, so the diagnosis was confirmed by spirometry, which demonstrated a $21 \%$ decrease in her force expiratory volume in 1 second after she handled sesame seeds for 15 minutes. $^{28}$

\section{Management Primary prevention}

The development of methods to prevent food allergy is a major area of clinical allergy research. One case-control study assessed the effect of sesame consumption during pregnancy, and one randomized controlled trial (RCT) evaluated the effect of early introduction in the first months of life.

In a case-control study of 1272 children, Hsu et al assessed whether maternal sesame seed protein exposure during pregnancy, in the form of progesterone formulated in sesame seed oil for luteal support or maternal consumption of sesame seeds, increased the risk of sensitization to peanuts, tree nuts, and/or sesame in the child. They found that progesterone oil formulation exposure was not associated with increased odds of the child being sensitized to peanut, tree nut, and/or sesame. Women who consumed sesame seeds during pregnancy were more likely to have a child that was sensitized to one or more of peanut, tree nut, and sesame (OR 1.78, 95\% CI 1.08-2.92 for sesame consumption during the first trimester), but whether the sensitized children had true food allergy was not confirmed. ${ }^{29}$ Maternal avoidance of allergenic foods during pregnancy is not currently recommended by allergy guideline societies. ${ }^{30,31}$

In a large RCT, the enquiring about tolerance (EAT) study assessed whether the introduction of sesame (as well as five other allergenic foods) at 3 months of age in exclusively breast-fed infants reduced the likelihood of developing sesame allergy compared to the introduction of sesame after 6 months of age. They concluded that there was no difference in the incidence of sesame allergy in the early and standard introduction groups after 3 years of follow-up. ${ }^{32}$ However, since the primary outcome of the EAT study evaluated allergy to one or more of six allergenic foods, not just sesame allergy, it was not sufficiently powered to detect a difference in the incidence of sesame allergy between the two groups. Furthermore, only $44 \%$ of patients were compliant with the early introduction regimen for sesame. ${ }^{33}$

\section{Allergen avoidance}

One of the mainstays of food allergy management is allergen avoidance. Successful avoidance requires patients to have accurate information on the ingredients and possible allergenic contaminants in food, and consequently several countries have developed regulations for food allergen labeling. The European Union, Australia, New Zealand, and Canada have listed sesame as one of the allergens for which there are food-labeling requirements. A notable exception is the USA where the current regulatory framework does not recognize sesame as a priority allergen. ${ }^{34}$

To study the prevalence of precautionary allergen labeling, an Australian study evaluated 1355 prepackaged products from one supermarket in Melbourne, Australia. They found that $6.1 \%$ of items listed sesame as an ingredient and $27.5 \%$ of items included a precautionary statement regarding sesame. Sesame was the third most common allergen listed on such precautionary labels. The most common types of food that included precautionary labeling for sesame were 
savory biscuits $(86 \%)$, bread products $(73.3 \%)$, and sweet biscuits $(71.1 \%) .35$

When educating patients about the avoidance of such foods, it may be helpful to describe the quantity of sesame seeds that could trigger a reaction in a highly sensitive patient. Since a sesame seed weighs $\sim 3.2 \mathrm{mg}$ (corresponding to $\sim 0.544 \mathrm{mg}$ of sesame protein) and the lowest reported LOAEL is $1.02 \mathrm{mg}$, just two sesame seeds could trigger an allergic reaction, ${ }^{27}$ supporting the need for careful allergen avoidance.

Despite strict food avoidance, accidental exposures can occur. In a Canadian study, the parents of 115 children with a known diagnosis of sesame allergy were surveyed on whether their children had experienced an accidental exposure. Accidental exposures were relatively common with an annual rate of $15.9 \%$. Older children were more likely to experience an accidental exposure, possibly due to increased risk-taking behaviors and less supervision. ${ }^{8}$ This is an important finding given that the development of tolerance to sesame appears to be relatively uncommon. In a cross-sectional study that surveyed parents of food allergic children, an American group showed that only $27.1 \%$ of sesame-allergic children achieved tolerance. ${ }^{9}$ Cohen et a ${ }^{10}$ similarly noted that $20 \%$ developed tolerance in their cohort after a mean follow-up length of 6.7 years and an Israeli group found that $30 \%$ of sesame-allergic children achieved tolerance after a mean follow-up time of 4.7 years. ${ }^{36}$

\section{Epinephrine autoinjector (EAI)}

Given that the first-line treatment in case of an accidental reaction is prompt epinephrine administration, patients with a sesame allergy must keep an EAI available at all times, recognize the signs and symptoms of anaphylaxis, and reliably self-administer the medication when necessary (or have a parent do so if the patient is a young child). However, the Canadian study discussed earlier showed that $<10 \%$ of sesame-allergic patients who experienced an accidental exposure used their EAI. Equally worrisome was that of the 49 patients who already possessed an EAI prior to their initial reaction to sesame, only 6 used the device. ${ }^{8}$

The prescription of an EAI should not be considered a benign intervention. Pinczower et al found in a crosssectional study of 103 children, 17 of whom had sesame allergy, that prescription of EAI negatively affected healthrelated quality of life. When required, the prescription of an EAI should be accompanied by patient education with regular reinforcement. ${ }^{37}$

\section{Complementary and alternative medicine}

Traditional Chinese medicine (TCM) has attracted interest in Western countries as a source of alternative medicine for a variety of diseases, including food allergy. The most extensively studied drug for food allergy that originated from TCM is Food Allergy Herbal Formula-2 (FAHF-2), a nine-herb formula based on the traditional Chinese formula Wu Mei Wan. As previous phase 1 trials had demonstrated its safety and murine models had shown encouraging immunomodulatory effects, an RCT was undertaken to assess its efficacy. A total of 58 subjects with food allergy, including sesame, underwent a baseline DBPCFC and a second DBPCFC after 6 weeks of treatment with FAHF-2 to assess for an increase in the dose of allergen eliciting a reaction. FAHF-2 was not found to be efficacious, however, as significantly more subjects treated with placebo compared to FAHF-2 had improvements in consumed allergen dose at the follow-up DBPCFC, though its safety and beneficial in-vitro immunomodulatory effects were again demonstrated. Subgroup analysis for the sesameallergic group was not published..$^{38}$

\section{Future directions Basophil activation testing (BAT)}

BAT is a novel in-vitro diagnostic tool with emerging applications in food allergy diagnosis. Stimulation of basophils with allergen to which they are sensitive induces the expression of cell-surface proteins, and these proteins can be detected with specific antibodies using flow cytometry. CD63 is the most common marker under study, though several other candidate markers have been identified. ${ }^{39}$ Studies evaluating the use of BAT in the diagnosis of sesame allergy are highly limited. A German group reported a case of a 47-year-old male with suspected sesame allergy who had a negative SPT, sIgE, and SPP testing to sesame seeds and paste. BAT was strongly positive, however, and sesame allergy was confirmed by DBPCFC, suggesting a role for BAT in the diagnosis of sesame allergy. ${ }^{40}$ In a study of a mixed group of patients with one or more food allergies, including sesame, BAT was found to have better diagnostic accuracy compared to sIgE with an AUC of 0.904 compared to 0.870 and was correlated with DBPCFC severity scores. However, only 6 of the 67 patients enrolled were sensitized to sesame, so specific conclusions regarding the use of BAT in the diagnosis of sesame allergy were not possible. ${ }^{41}$ Methods for using BAT with sesame protein components are also under development but remain experimental. ${ }^{42}$ 


\section{Immunotherapy}

Oral and sublingual allergen-specific immunotherapies have been proposed as possible disease-modifying treatments for food allergy. Most such immunotherapy protocols involve the provision of progressively higher doses of the allergen with the goal of modulating the immune response to the allergen. If desensitization is achieved, patients can eat larger quantities of the allergen without triggering a reaction, but they must consume the allergen daily. If true tolerance is achieved, a state of sustained unresponsiveness develops in which the patient can consume usual quantities of the food intermittently with no reactions. Studies have shown some success using these methods with food allergens such as milk, peanut, and egg, but up to this point the efficacy of sesame immunotherapy has never been systematically studied. Bégin et al, however, demonstrated the safety and feasibility of oral immunotherapy using multiple food allergens simultaneously in 25 patients, 6 of whom were sesame-allergic and received sesame immunotherapy. All the patients who completed the study achieved the target 10 -fold increase in reaction threshold, though as a phase 1 trial, the study was not designed for the evaluation of efficacy. ${ }^{43}$

\section{Conclusion}

The diagnosis of sesame allergy currently relies on the clinical history supplemented by testing for sesame sensitization and OFC in certain cases. SPT appears to be more informative compared to $\operatorname{sgE}$ in children and adults, though data in adults remain highly limited. Novel diagnostic tools including component-resolved diagnostics and BAT for the diagnosis of sesame allergy are also under development. The mainstays of management include the prescription of an EAI and patient education regarding allergen avoidance and prompt use of epinephrine in case of an accidental reaction. The induction of tolerance by immunotherapy is an attractive management option that is under investigation. Future studies of novel diagnostic methods and management techniques will transform how allergy to sesame, a major allergen of increasing importance, is approached in the years ahead.

\section{Disclosure}

The authors report no conflicts of interest in this work.

\section{References}

1. Sicherer SH, Muñoz-Furlong A, Godbold JH, Sampson HA. US prevalence of self-reported peanut, tree nut, and sesame allergy: 11-year follow-up. J Allergy Clin Immunol. 2010;125(6):1322-1326.

2. Ben-Shoshan M, Harrington DW, Soller L, et al. A population-based study on peanut, tree nut, fish, shellfish, and sesame allergy prevalence in Canada. J Allergy Clin Immunol. 2010;125(6):1327-1335.
3. Soller L, Ben-Shoshan M, Harrington DW, et al. Adjusting for nonresponse bias corrects overestimates of food allergy prevalence. JAllergy Clin Immunol Pract. 2015;3(2):291-293.

4. Osborne NJ, Koplin JJ, Martin PE, et al; HealthNuts Investigators. Prevalence of challenge-proven IgE-mediated food allergy using population-based sampling and predetermined challenge criteria in infants. J Allergy Clin Immunol. 2011;127(3):668-676.e1-2.

5. Dalal I, Binson I, Reifen R, et al. Food allergy is a matter of geography after all: sesame as a major cause of severe IgE-mediated food allergic reactions among infants and young children in Israel. Allergy. 2002;57(4):362-365.

6. Sheikh F, Amin R, Rehan Khaliq AM, Al Otaibi T, Al Hashim S, Al Gazlan S. First study of pattern of anaphylaxis in a large tertiary care hospital in Saudi Arabia. Asia Pac Allergy. 2015;5(4):216.

7. Ben-Shoshan M, La Vieille S, Eisman H, et al. Anaphylaxis treated in a Canadian pediatric hospital: incidence, clinical characteristics, triggers, and management. J Allergy Clin Immunol. 2013;132(3):739-741.e3.

8. Segal L, Ben-Shoshan M, Alizadehfar R, et al. Initial and accidental reactions are managed inadequately in children with sesame allergy. J Allergy Clin Immunol Pract. 2017;5(2):482-485.

9. Gupta RS, Lau CH, Sita EE, Smith B, Greenhawt MJ. Factors associated with reported food allergy tolerance among US children. Ann Allergy Asthma Immunol. 2013;111(3):194-198.e4

10. Cohen A, Goldberg M, Levy B, Leshno M, Katz Y. Sesame food allergy and sensitization in children: the natural history and long-term followup. Pediatr Allergy Immunol. 2007;18(3):217-223.

11. Peters RL, Allen KJ, Dharmage SC, et al; HealthNuts Study. Skin prick test responses and allergen-specific $\mathrm{IgE}$ levels as predictors of peanut, egg, and sesame allergy in infants. JAllergy Clin Immunol. 2013;132(4): 874-880.

12. Permaul P, Stutius LM, Sheehan WJ, et al. Sesame allergy: role of specific IgE and skin-prick testing in predicting food challenge results. Allergy Asthma Proc. 2009;30(6):643-648.

13. Stutius LM, Sheehan WJ, Rangsithienchai P, et al. Characterizing the relationship between sesame, coconut, and nut allergy in children: sesame and coconut allergy in children. Pediatr Allergy Immunol. 2010;21(8): $1114-1118$.

14. Leduc V, Moneret-Vautrin DA, Tzen JTC, Morisset M, Guerin L, Kanny G. Identification of oleosins as major allergens in sesame seed allergic patients. Allergy. 2006;61(3):349-356.

15. Della-Torre E, Pignatti P, Yacoub M-R, Sabbadini M-G, Colombo G. In vivo tests with "Tahini" sauce: new allergenic source to evaluate IgE-mediated hypersensitivity to sesame. Ann Allergy Asthma Immunol. 2013;110(3):209-210.

16. Alonzi C, Campi P, Gaeta F, Pineda F, Romano A. Diagnosing IgEmediated hypersensitivity to sesame by an immediate-reading "contact test" with sesame oil. J Allergy Clin Immunol. 2011;127(6):1627-1629.

17. Foong RM, Logan K, Fox AT, Du Toit G. Clinical characteristics of-and predictive diagnostic factors for-sesame seed allergy in food-allergic children: original research. Curr Allergy Clin Immunol. 2013;26(2): 78-81.

18. Barbarroja-Escudero J, Sanchez-Gonzalez M-J, Antolin-Amerigo D, Rodriguez-Rodriguez M, Pineda F, Alvarez-Mon M. Diagnosis of IgE-mediated hypersensitivity to sesame seeds supplemented with lipid body proteins. Allergol Int. 2015;64(4):396-398.

19. Zavalkoff S, Kagan R, Joseph L, St-Pierre Y, Clarke A. The value of sesame-specific IgE levels in predicting sesame allergy. J Allergy Clin Immunol. 2008;121(6):1508-1510.

20. Maloney JM, Rudengren M, Ahlstedt S, Bock SA, Sampson HA. The use of serum-specific IgE measurements for the diagnosis of peanut, tree nut, and seed allergy. J Allergy Clin Immunol. 2008;122(1):145-151.

21. Tuano KTS, Dillard KH, Guffey D, Davis CM. Development of sesame tolerance and cosensitization of sesame allergy with peanut and tree nut allergy in children. Ann Allergy Asthma Immunol. 2016;117(6): 708-710.

22. Maruyama N, Nakagawa T, Ito K, et al. Measurement of specific IgE antibodies to Ses 11 improves the diagnosis of sesame allergy. Clin Exp Allergy. 2016;46(1):163-171. 
23. Teodorowicz M, Terlouw RJ, Jansen A, Savelkoul HF, RuinemansKoerts J. Immunological characterization of Dutch sesame seed-allergic patients. Int Arch Allergy Immunol. 2016;169(1):13-22.

24. Asero R, Cecchi L, Cervone M, et al. Detection of $20 \mathrm{kDa}$ and $32 \mathrm{kDa}$ IgE-binding proteins as the major allergens in Italian sesame seed allergic patients. Eur Ann Allergy Clin Immunol. 2014;46(1):22-25.

25. Magni C, Ballabio C, Restani $\mathrm{P}$, et al. Molecular insight into IgEmediated reactions to sesame (Sesamum indicum L.) seed proteins. Ann Allergy Asthma Immunol. 2010;105(6):458-464.

26. Taylor SL, Baumert JL, Kruizinga AG, et al; Allergen Bureau of Australia \& New Zealand. Establishment of reference doses for residues of allergenic foods: report of the VITAL expert panel. Food Chem Toxicol. 2014;63:9-17.

27. Dano D, Remington BC, Astier C, et al. Sesame allergy threshold dose distribution. Food Chem Toxicol. 2015;83:48-53.

28. Caimmi S, Marseglia A, Caimmi D, Marseglia GL. Friday asthma crisis in the daughter of two bakers. Int J Immunopathol Pharmacol. 2011;24(2):517-518

29. Hsu JT, Missmer SA, Young MC, et al. Prenatal food allergen exposures and odds of childhood peanut, tree nut, or sesame seed sensitization. Ann Allergy Asthma Immunol. 2013;111(5):391-396.

30. Fleischer DM, Spergel JM, Assa'ad AH, Pongracic JA. Primary prevention of allergic disease through nutritional interventions. J Allergy Clin Immunol Pract. 2013;1(1):29-36.

31. Chan ES, Cummings C, Atkinson A, et al. Dietary exposures and allergy prevention in high-risk infants: a joint position statement of the Canadian Society of Allergy and Clinical Immunology and the Canadian Paediatric Society. Allergy Asthma Clin Immunol. 2014;10(1):1.

32. Perkin MR, Logan K, Tseng A, et al. Randomized trial of introduction of allergenic foods in breast-fed infants. N Engl J Med. 2016;374(18): 1733-1743.
33. Perkin MR, Logan K, Marrs T, et al; EAT Study Team. Enquiring about tolerance (EAT) study: feasibility of an early allergenic food introduction regimen. J Allergy Clin Immunol. 2016;137(5):1477-1486.e8.

34. Gendel SM. Comparison of international food allergen labeling regulations. Regul Toxicol Pharmacol. 2012;63(2):279-285.

35. Zurzolo GA, Mathai ML, Koplin JJ, Allen KJ. Precautionary allergen labelling following new labelling practice in Australia: precautionary allergen labelling. J Paediatr Child Health. 2013;49(4):E306-E310.

36. Aaronov D, Tasher D, Levine A, Somekh E, Serour F, Dalal I. Natural history of food allergy in infants and children in Israel. Ann Allergy Asthma Immunol. 2008;101(6):637-640.

37. Pinczower GD, Bertalli NA, Bussmann N, et al. The effect of provision of an adrenaline autoinjector on quality of life in children with food allergy. J Allergy Clin Immunol. 2013;131(1):238-240.e1.

38. Wang J, Jones SM, Pongracic JA, et al. Safety, clinical, and immunologic efficacy of a Chinese herbal medicine (Food Allergy Herbal Formula-2) for food allergy. J Allergy Clin Immunol. 2015;136(4):962-970.e1.

39. MacGlashan DW. Basophil activation testing. J Allergy Clin Immunol. 2013;132(4):777-787.

40. Raap U, Wieczorek D, Schenck F, Kapp A, Wedi B. The basophil activation test is a helpful diagnostic tool in anaphylaxis to sesame with false-negative specific IgE and negative skin test. Allergy. 2011;66(11):1497-1499.

41. Song Y, Wang J, Leung N, et al. Correlations between basophil activation, allergen-specific IgE with outcome and severity of oral food challenges. Ann Allergy Asthma Immunol. 2015;114(4):319-326.

42. Wallowitz ML, Chen RJY, Tzen JTC, Teuber SS. Ses i 6, the sesame $11 \mathrm{~S}$ globulin, can activate basophils and shows cross-reactivity with walnut in vitro. Clin Exp Allergy. 2007;37(6):929-938.

43. Bégin P, Winterroth LC, Dominguez T, et al. Safety and feasibility of oral immunotherapy to multiple allergens for food allergy. Allergy Asthma Clin Immunol. 2014;10(1):1.
Journal of Asthma and Allergy

\section{Publish your work in this journal}

The Journal of Asthma and Allergy is an international, peer-reviewed open access journal publishing original research, reports, editorials and commentaries on the following topics: Asthma; Pulmonary physiology; Asthma related clinical health; Clinical immunology and the immunological basis of disease; Pharmacological interventions and

\section{Dovepress}

new therapies. This journal is included in PubMed. The manuscript management system is completely online and includes a very quick and fair peer-review system, which is all easy to use. Visit http://www. dovepress.com/testimonials.php to read real quotes from published authors. 